\title{
Editorial
}

\section{D Nanomaterials: Synthesis, Properties, and Applications}

\author{
Yun Zhao, ${ }^{1}$ Haiping Hong, ${ }^{2}$ Qianming Gong, ${ }^{3}$ and Lijun $\mathrm{Ji}^{4}$ \\ ${ }^{1}$ School of Chemical Engineering and Environment, Beijing Institute of Technology, Beijing 100081, China \\ ${ }^{2}$ Department of Materials and Metallurgical Engineering, South Dakota School of Mines and Technology, Rapid City, SD 57701, USA \\ ${ }^{3}$ Department of Mechanical Engineering, Tsinghua University, Beijing 100084, China \\ ${ }^{4}$ College of Chemistry and Chemical Engineering, Yangzhou University, Yangzhou, Jiangsu 225009, China
}

Correspondence should be addressed to Yun Zhao; zhaoyun@bit.edu.cn

Received 12 June 2013; Accepted 12 June 2013

Copyright ( 2013 Yun Zhao et al. This is an open access article distributed under the Creative Commons Attribution License, which permits unrestricted use, distribution, and reproduction in any medium, provided the original work is properly cited.

One-dimensional (1D) nanostructured materials, including nanotubes, nanofibers, and nanowires, have attracted much attention due to their interesting properties and wide range of potential applications. Considerable research has been conducted on new routes to controllable synthesis of 1D nanomaterials. The unique properties of as-obtained 1D nanomaterials can lead to applications in various fields such as electronics, magnetism, optics, and catalysis. Therefore, researchers working in the $1 \mathrm{D}$ nanomaterials are constantly striving to develop new fundamental science as well as potential applications. The importance of a platform that allows active researchers in this field to present their new development in a timely and efficient manner is, therefore, necessary.

This special issue focuses on the recent progress in production techniques, novel properties, and applications of various 1D nanomaterials including oxides/metals/nitrides with 1D nanostructures, carbon nanotubes, and nanofibers.

A total of 11 articles are presented in the current issue and they are all research papers. They involve spin transport properties of AlN nanowires, photoluminescence of $\mathrm{ZnO}$ nanorods, heating efficiency of iron nanowires, optical properties and thermal stability of poly(vinyl alcohol) composite nanofibers, electrical properties of half-doped manganite single nanowire, carbon nanotube yarns, and pristine and functional single-wall carbon nanotubes. Production techniques include a hydrothermal reaction, reducing method, chemical vapor deposition, spinning method, and microemulsion system. Carbon nanotubes have attracted most research interest, maintaining their comfortable leading positions.
We are pleased to see the progress in synthesis and properties of 1D nanomaterials. We hope that this special issue will promote further development of large-scale economically feasible 1D nanomaterial-making technologies and also contribute to their wide use.

\section{Acknowledgments}

The editors gratefully thank the authors for their contributions to this special issue and the reviewers for their constructive comments and dedication.

Yun Zhao Haiping Hong Qianming Gong Lijun Ji 

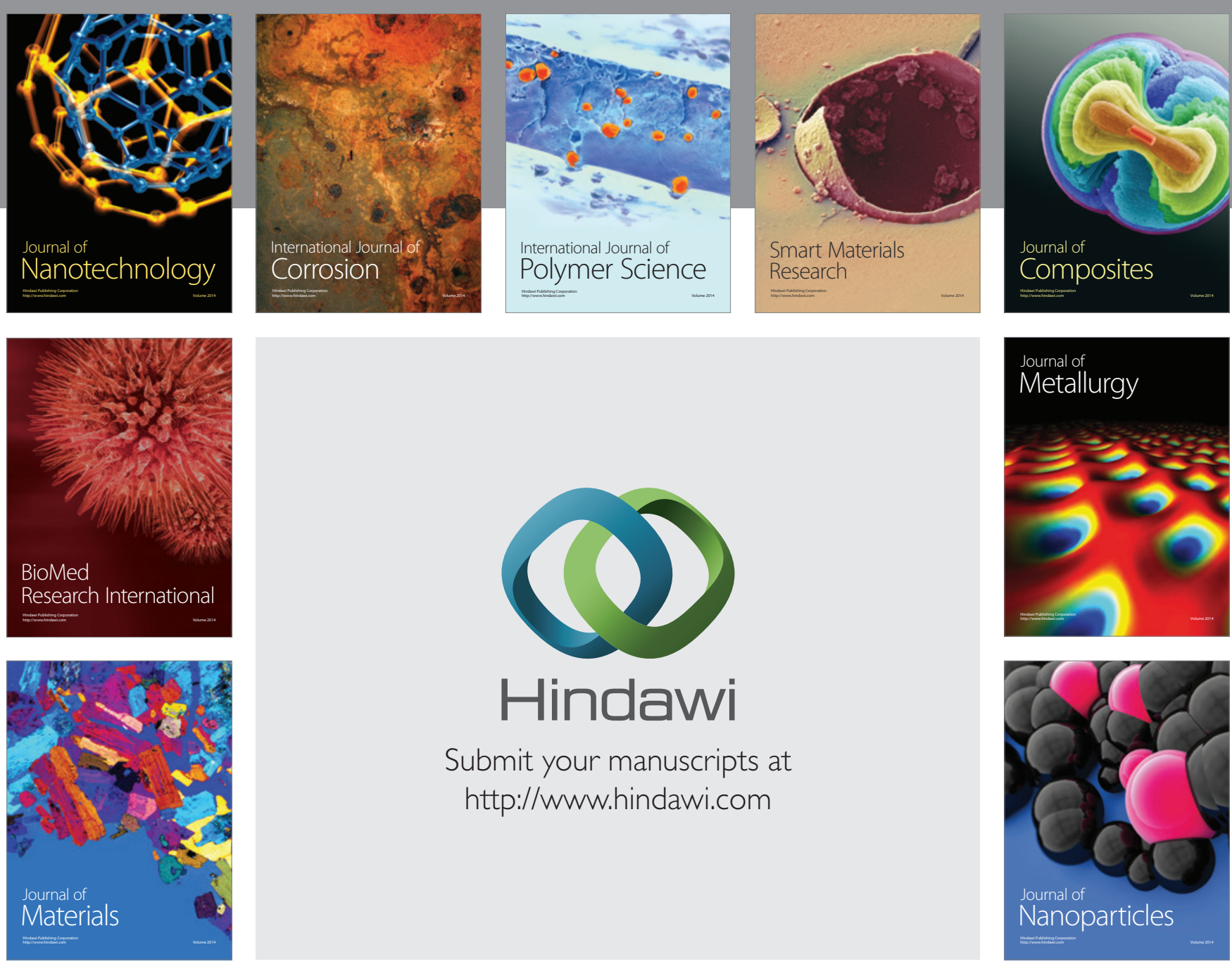

Submit your manuscripts at http://www.hindawi.com
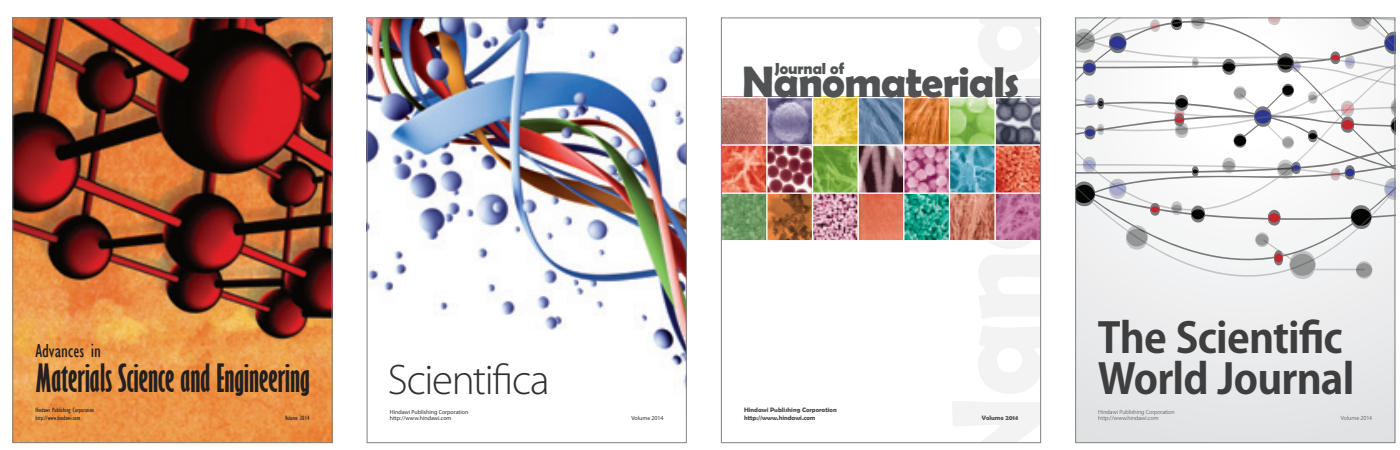

\section{The Scientific World Journal}
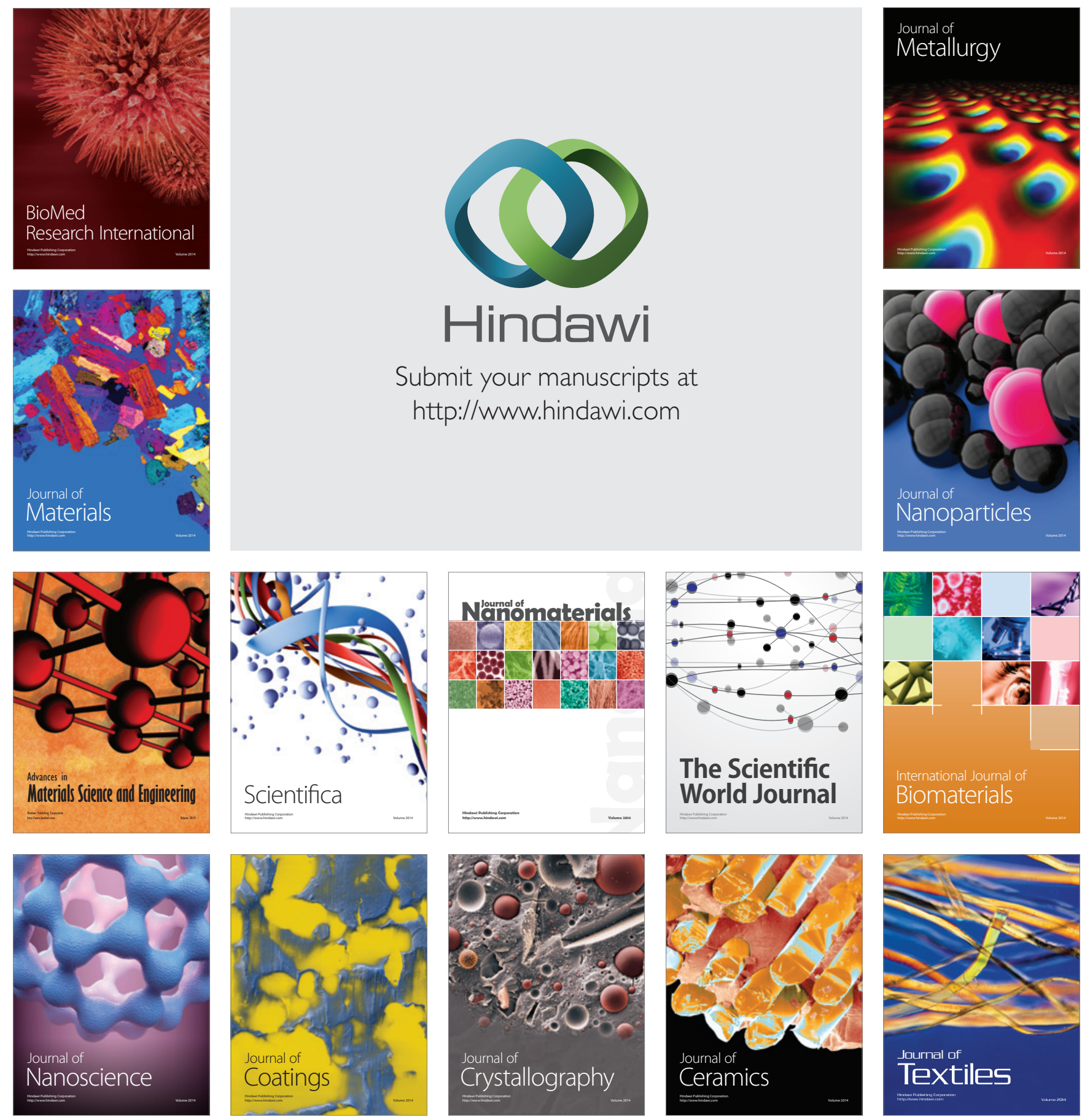